\title{
Adherence to the American Society for Colposcopy and Cervical Pathology Guidelines: An Observational Study
}

\author{
Sarah Conrad ${ }^{1}$, Bassam Dahman ${ }^{1},{\text { Christine } \text { Isaacs }^{1} \text {, Anita Kumar }}^{1}$, and Jordan Hylton ${ }^{1}$ \\ ${ }^{1}$ Virginia Commonwealth University
}

December 24, 2020

\begin{abstract}
Objective: To evaluate adherence to the 2012 ASCCP guidelines by physicians referring patients to a large academic center for a colposcopy and to understand the factors associated with incorrect referrals. Design: A retrospective observational study Setting: A large tertiary referral hospital Population: Woman referred to Virginia Commonwealth University for colposcopy or loop electrosurgical excision procedure from January 2015 to December 2016. Methods: Data on patient demographics, cervical cytology, human papillomavirus status, and the recommended interventions were gathered. Main Outcome Measures: Concordance with ASCCP guidelines Results: Referral requests for 430 women were reviewed. Of the referrals, $17.4 \%$ were discordant with the ASCCP guidelines. The most common discordant colposcopy referrals were for low-grade squamous intraepithelial (LSIL) lesion (48\%) and atypical squamous cell of undetermined significance (29\%). The likelihood of incorrect referral was decreased for high grade lesions (OR 0.03), increased in women age $<25$ (OR 31.6) and those referred by family medicine (OR 3.6) or internal medicine (OR 4.4). Ten patients were referred for cervical cytology collected on vaginal cuffs despite hysterectomies performed for benign reasons. Conclusions: Patients referred outside of the guidelines were most often women age $<25$ with low-grade lesions. Referrals outside of evidence-based guidelines may lead to unnecessary procedures and added healthcare expense. Our results help identify areas for provider education and potential areas for concern in the implementation of the 2019 ASCCP updates.
\end{abstract}

Title: Adherence to the American Society for Colposcopy and Cervical Pathology Guidelines: An Observational Study

Authors: Sarah Conrad ${ }^{1}$ MD, Bassam Dahman ${ }^{2}$ PhD, Anita Kumar ${ }^{2}$, Jordan Hylton ${ }^{1}$ MD, Christine Isaacs $^{1} \mathrm{MD}$

${ }^{1}$ Virginia Commonwealth University, Richmond, Virginia, United States. Department of Obstetrics and Gynecology

${ }^{2}$ Virginia Commonwealth University, Richmond, Virginia, United States. Department of Health Behavior and Policy

Short Title: Adherence to the 2012 ASCCP Guidelines

\section{Corresponding Author:}

Sarah Kay Conrad.

P.O. Box 980034 Richmond, VA 23298 United States

Work: (804) 828-8614

Cell: 713-417-6505

Home: 713-417- 6505 


\begin{abstract}
Objective: To evaluate adherence to the 2012 ASCCP guidelines by physicians referring patients to a large academic center for a colposcopy and to understand the factors associated with incorrect referrals. Design: A retrospective observational study
\end{abstract}

Setting: A large tertiary referral hospital

Population: Woman referred to Virginia Commonwealth University for colposcopy or loop electrosurgical excision procedure from January 2015 to December 2016.

Methods : Data on patient demographics, cervical cytology, human papillomavirus status, and the recommended interventions were gathered.

Main Outcome Measures: Concordance with ASCCP guidelines

Results: Referral requests for 430 women were reviewed. Of the referrals, $17.4 \%$ were discordant with the ASCCP guidelines. The most common discordant colposcopy referrals were for low-grade squamous intraepithelial (LSIL) lesion (48\%) and atypical squamous cell of undetermined significance (29\%). The likelihood of incorrect referral was decreased for high grade lesions (OR 0.03), increased in women age $<25$ (OR 31.6) and those referred by family medicine (OR 3.6) or internal medicine (OR 4.4). Ten patients were referred for cervical cytology collected on vaginal cuffs despite hysterectomies performed for benign reasons.

Conclusions: Patients referred outside of the guidelines were most often women age $<25$ with low-grade lesions. Referrals outside of evidence-based guidelines may lead to unnecessary procedures and added healthcare expense. Our results help identify areas for provider education and potential areas for concern in the implementation of the 2019 ASCCP updates.

Keywords: ASCCP Guidelines, colposcopy, pap test, LEEP, cervical cancer, cervical dysplasia, CIN

Tweetable Abstract: $17.4 \%$ of colposcopy referrals to a large academic center were discordant with the ASCCP guidelines.

\title{
Main Text
}

\section{Introduction}

Cervical cancer screening and colposcopy have played an integral role in reducing the prevalence of cervical cancer over the past 40 years (1). The guidelines for cervical cancer screening and colposcopy were established more than 15 years ago by the American Society for Colposcopy and Cervical Pathology (ASCCP) and modified over the years as a broader understanding of cervical cancer screening has increased (2). While all women are at risk for cervical cancer, women over the age of 30 are at a greater risk of developing cervical cancer (1). Furthermore, the majority of human papillomavirus (HPV) in women under the age of 24 resolve spontaneously within one to three years (3). The 2012 ASCCP guidelines were developed to identify cervical pathology as well as minimize overtreatment of lesions that may resolve spontaneously. Colposcopy and loop electrosurgical excision procedures (LEEP) allow for the identification and treatment of pre-invasive lesions, and aid in early detection of invasive cervical lesions when treatment is more effective (4). Unnecessary colposcopy procedures may result in increased cost, unnecessary treatment, and serious psychological consequences for women (5). Additionally, the 2019 ASCCP guidelines have transitioned from result-based algorithms to risk based (6).

There have been several studies that evaluated the adherence of health care providers to the 2012 guidelines in terms of correct screening intervals and knowledge of HPV co-testing $(7,8)$. According to Teoh et all, $15 \%$ of providers were unaware of 2012 guideline changes however, this study did not report on adherence to guidelines regarding referring abnormal cytology results for colposcopy (7).

Virginia Commonwealth University (VCU) Department of Obstetrics and Gynecology 
conducts diagnostic procedures such as colposcopies for referred patients. The gynecology trained faculty physicians at VCU review all referrals and based on the referred cytology and HPV results make recommendations for subsequent care. The objectives of this study include evaluation of adherence to the 2012 ASCCP guidelines by outside physicians referring patients to a large academic center for a colposcopy and by the large academic center physician's recommendations following a colposcopy procedure, as well as, the identification of factors associated with referrals that are considered non-adherent to the 2012 ASCCP guidelines. An understanding of the factors associated with incorrect referrals for the 2012 ASCCP guidelines allows for identification of potential areas for concern in the implementation of the 2019 ASCCP guideline updates.

\section{Methods}

A retrospective study was performed evaluating the women referred to VCU for colposcopy or LEEP from January 2015 to December 2016. Electronic health records of 430 patients referred to the VCU clinic were utilized to identify patient demographics, cervical cytology, HPV status, prior cervical dysplasia, type of referring provider, patient show rate, the length of time from when the ASCCP guidelines were published and when the referral was received, and the recommended intervention. Except in specific clinical scenarios, pap tests are not recommended until 21 years of age and many women after the age of 65 stop receiving screening for cervical cancer therefore patients under the age of 21 and over the age of 65 were excluded from the study. The final study population consisted of 430 patients. The institutional review board approved this study (IRB HM2004659) as exempt on March 3, 2018, as it was a review of already existing information and posed minimal/no risk to subjects. Figure 1 is a pictorial reference of the methods process. All p-values less than 0.05 are considered statistically significant.

\section{Study Variables}

The ASCCP online application as well as the published updated guidelines were used to determine if patients were referred correctly and if the correct recommendation was then made by the receiving VCU gynecology provider (2). The primary outcome was if the referral was concordant with guidelines. A secondary outcome variable of interest was if the receiving VCU gynecology provider made the correct post-colposcopy recommendation that is concordant with the guidelines. The main explanatory variable is cervical cytology from pap-test results which is categorical in nature (atypical squamous cell of undetermined significance (ASCUS), Atypical squamous cells, cannot exclude high-grade squamous intraepithelial lesion

(ASCH), low-grade squamous intraepithelial lesion (LSIL), high-grade squamous intraepithelial lesion (HSIL) , Atypical Glandular Cells (AGC), and Negative for Intraepithelial Lesion or Malignancy (NILM)). Other variables representing adherence to the guidelines includes the VCU provider recommended procedure (colposcopy, LEEP, or re-screen) and HPV test results. Other covariates include patient's race/ethnicity, patient's age, type of referring provider (gynecologist, family medicine, internal medicine, health departments, veteran affairs, others), prior cervical dysplasia, and if the patient was seen at the VCU clinic. A variable representing the length of time from when the ASCCP guidelines were published and when the referral was received was included to control for the effect of the amount of passed time since the implementation of the guidelines and a referral to the VCU clinic. It is anticipated that the greater the amount of time passed will result in fewer discordant referrals. Due to the small sample size and unbalanced frequencies within variable categories some variable categories were combined or not included in the adjusted model. The variables for HPV result and prior colposcopy had many patients which were categorized as not applicable, likely due to the age recommendations for HPV testing and colposcopies therefore, these variables were not included in the multivariable logistic regression.

\section{Descriptive Statistics}

Cross tabulations were used to estimate the frequency and percent for the categories of all study variables. Chi-Square tests were used to determine the significance of the difference in the distributions of those variable between the concordant and discordant referrals. Chi-Square statistical analysis was used to estimate if there were statistically significant differences in cervical cytology results by age group for the covariates among the discordant referrals by cervical cytology and age. 


\section{Univariate Logistic Regression}

Univariate logistic regression was used to estimate the odds ratio and statistical significance of discordant referrals as a function of cervical cytology and the other covariates individually.

\section{Multivariable Logistic Regression}

To adjust for confounders a multivariable logistic regression analysis was used. The model estimated the likelihood and statistical significance of a discordant referral as a function of the cervical cytology while controlling for clinic type, race, age group, and time between policy and referral to the VCU Department of Obstetrics and Gynecology. We used the c-statistic to evaluate the strength of the model's ability in predicting similar outcomes to those observed in the study population.

\section{Results}

\section{Descriptive Statistics}

Descriptive statistics which are included in table one indicate that out of the 430 patients included in the study sample, there were $355(82.56 \%)$ concordant referrals and $75(17.44 \%)$ discordant referrals. The referrals resulted in recommendations by the VCU physician for $340(79.07 \%)$ colposcopies, $57(13.02 \%)$ re-screen tests, and 35 (7.91\%) LEEP procedures. The cervical cytology with the most discordant referrals included pap results for ASCUS (29\%), LSIL (48\%), and NILM (20\%). The VCU attending physician reviewing the referrals had a $95 \%$ adherence rate to the 2012 ASCCP guidelines. The VCU reviewing attending was able to identify $72 \%$ of the discordant referrals as non-adherent to the 2012 ASCCP guidelines. There were 18 (4.17\%) patients that following colposcopies did not receive a recommendation for follow-up testing from the VCU physician that was concordant with the 2012 ASCCP guidelines. Six (33\%) of these 18 patients had already discordant referrals for a colposcopy and then were given follow-up testing recommendations by VCU physicians that were not concordant with the 2012 ASCCP guidelines. Family medicine providers and health departments comprised $40 \%$ and $28 \%$ of the discordant referrals respectively. Approximately $51 \%$ of the study population was African American, whites comprised approximately $31 \%$, and $11 \%$ of the study population was Hispanic. All variables had statistically significant differences between concordant and discordant referrals.

(please see table 1)

Table 2 further separates discordant referrals by age group. For patients between 21 and 24 years of age, there were 31 (41\%) discordant referrals. Of which 6 (19\%) had pap-test results indicating ASCUS, 24 (77\%) had LSIL results, and $1(0.3 \%)$ had a NILM pap-test result. For discordant referrals in this age range there were significant differences for HPV results among the cervical cytology categories however, there were not any statistically significant differences in other variables.

In the 25 to 29 age range, there were 5 discordant referrals, one with a pap-test result of ASCUS and the other 4 with NILM pap-test result. There were $39(52 \%)$ patients in the 30 to 64 age range with discordant referrals with $38 \%$ with ASCUS results, $31 \%$ with LSIL results, $5 \%$ with HSIL results, and $26 \%$ with NILM pap-test results.

Not included in the table, of the discordant LSIL in patients age 21 to 24 , seven went on to receive nonindicated colposcopies at VCU. Furthermore, two of these patients underwent a LEEP procedure as the colposcopy for LSIL at age 21 to 24 resulted CIN II or CIN III. The most common discordance in regards to ASCUS referrals was when the co-testing of high-risk HPV subtypes was negative $(\mathrm{n}=7)$. Seven women age 21 to 24 with ASCUS cytology and positive HPV were referred for colposcopy instead of repeat cytology in one year. Additionally, seven referrals for ASCUS cytology did not have any co-testing or reflex testing.

In addition to the mentioned findings, ten patients were referred for cervical cytology collected on vaginal cuffs despite prior hysterectomies performed for benign reasons. Two of these had findings of HSIL. Additionally, seven patients were referred with the first occurrence of HPV+ screening with NILM cytology instead of having co-testing in twelve months. 


\section{Simple and Multivariable Logistic Regression}

When controlling for other covariates the adjusted model resulted in a decreased likelihood of a discordant referral for those with high-grade lesions (ASCH/AGC/HSIL) (OR: 0.03; CI: 0.006, 0.166; p<0.001) compared to those with low grade or no lesions (ASCUS/LSIL/NILM) results. These results suggest that those with low-grade lesions are 97 times more likely to be incorrectly referred for a colposcopy. The adjusted models' odds of a discordant referral with high-grade lesions was much lower than the univariate regression for the odds of a discordant referral with high-grade lesions (OR: 0.75; CI: $0.58,0.96 ; \mathrm{p}<0.05$ ). The likelihood of a discordant referral from a family medicine provider (OR:7.72; CI: 2.23, 26.69; $\mathrm{p}<0.01$ ) and internal medicine provider (OR:10.94; CI: 2.56, 46.79; p<0.01) compared to an OBGYN is also much higher in the adjusted model compared to the likelihood of a discordant referral from a family medicine provider (OR:4.12; CI:1.58,10.74; $\mathrm{p}<0.01)$ and internal medicine (OR:5.42; CI:1.63,18.03; $\mathrm{p}<0.01)$ physician in the unadjusted model. The likelihood of a discordant referral in patients under 24 years of age (OR: 19.55; CI: 7.62,50.15; $\mathrm{p}<0.001)$ compared to women between the ages of 25 and 29 is much higher in the adjusted model compared to women under the age of 24 (OR:7.80; CI:4.09,14.86; p<0.001) in the unadjusted model. The multivariate logistic model strongly predicted similar rates of correct referrals to those observed in the sample (c-statistic $0.873)$.

(please see table 3)

The show rate for patients correctly referred from outside institutions for colposcopy or LEEP was $83.2 \%$.

\section{Discussion and Conclusions}

Our study demonstrates that $17.4 \%$ of referrals for colposcopy to a tertiary academic center were discordant with the 2012 ASCCP guidelines. Patients referred outside of the guidelines were more likely to be women age 21 to 24 or 30 to 64 with low grade lesions (e.g. ASCUS, LSIL, NILM). Based on our results patients age 25-29 are rarely incorrectly referred. This likely reflects the simpler guidelines in the age 25-29 group as HPV only comes into account when it is reflexed for ASCUS and LSIL lesions are referred regardless of HPV status. A patient receiving a screening test at a family medicine or internal medicine clinic is also more likely to be incorrectly referred. Furthermore, this study indicates that in our region the health departments trend towards following ASCCP recommendations most closely. African American patients comprised the majority of our patient population, but while not statistically significant trended towards likelihood of incorrect referrals when compared White or Hispanic patients. This finding may indicate underlying bias.

Multiple patients were also referred for cervical cytology collected on vaginal cuffs despite hysterectomies performed for benign reasons. In some cases, referrals outside of evidence-based guidelines led to non-indicated biopsies and unnecessary procedures. Our study also indicates that over a two year period there does not seem to be a change in referral accuracy.

Cervical cancer screening and appropriate referral for biopsy and or excision has greatly decreased the mortality from cervical cancer in the United States, (9) but the effectiveness of the ASCCP guidelines depends on provider knowledge and adherence. The 2012 guidelines extended adolescent guidelines to age 24 with the understanding that HPV infection is common in this age group and dysplastic lesions are most likely to resolve without intervention (4). Furthermore, infection with HPV is known to be necessary for the development of cervical cancer, and the 2012 guidelines specifically address how to approach discordant cytology screening results (e.g. HPV-/LSIL) (10,11). Additionally, primary vaginal cancer is very rare, and cytology collection is not recommended on vaginal cuffs for women who never had CIN 2 or higher when hysterectomy is performed for benign indications (12). Similar to prior studies that indicate lack of knowledge and adherence to screening intervals recommended by the ASCCP $(7,8)$. Our study suggests that there is also confusion regarding interpretation of cytology results specifically in women 21 to 24 and for discordant cytology and HPV results (e.g. HPV-/ LSIL). A survey administered in 2015 revealed that approximately $14 \%$ of advanced nurse practitioners were routinely collecting cytology on vaginal cuffs and $8 \%$ were unsure if patients who had a hysterectomy for benign reasons should have screening cytology (8). As shown in our study, patients with prior hysterectomies are not only incorrectly screened, but also being 
referred for un-indicated procedures

Future research in this field could include review of all cytology tests to identity patients that should have been referred for colposcopy, but were instead returned to screening. Future research could also include a survey to identify whether providers were aware of colposcopy guideline changes, the availability of a mobile application for interpretation and guidelines as well as a knowledge questionnaire of current guidelines. We could also expand data collection to include referrals from 2013 -current and again evaluate if there has been any change over time in referral concordance. Furthermore, guideline changes in cervical cancer screening have been published in 2020, education and vigilance to evidence-based algorithms must continue to be an important focus.

To our knowledge no other study has evaluated the adherence to referral guidelines for biopsy or excision. The strengths of this study include the large number of referrals reviewed and as shown in Table 1, the varied clinical subspecialties that referred to a large academic center for colposcopy and LEEP. This study is limited in that it does not identify patients with pathology that should have warranted a referral but were returned to routine screening.

Our study indicates that overall the majority of referrals to a tertiary center were concordant with published guidelines, however there is room for improvement, specifically for cytology results in the patients age $<25$. Our results also indicate that some providers, especially in fields outside of gynecology may not take into account the necessity of HPV for the development of cervical cancer when referring patients for colposcopy, or the lack of need for screening in the context of hysterectomy performed for benign reasons. Referrals outside of evidence-based guidelines may lead to unnecessary procedures especially of the reproductive age patient, increased patient stress and added healthcare expense.

The 2019 ASCCP guidelines have transitioned from results- based algorithms to risk based algorithms. However in the age group 25 and under the algorithms have been carried forward (6). Our results indicated that in this age group where HPV has a high probability for regression, patients were incorrectly referred. Future studies could evaluate referral concordance overtime, especially in the less than 25 age group. Future research could also include surveys to providers that incorrectly refer and whether they use the updated ASCCP application, especially as the application is not free of charge.

\section{Acknowledgements}

Elizabeth King, $\mathrm{MD}^{2}$ for her assistance in data collection.

${ }^{2}$ Present Address. Mayo Clinic School of Graduate Medical Ed, Rochester, Minnesota. Department of Pediatrics

\section{Disclosure of Interests}

Dr. Sarah Conrad reports no competing financial interests

Dr. Christine Isaacs reports no competing financial interests

Dr. Jordan Hylton reports no competing financial interests

Dr. Bassam Dahman reports no competing financial interests

Anita Kumar reports no competing financial interests

\section{Contribution to Authorship}

$\mathrm{SC}, \mathrm{JH}$ and $\mathrm{CI}$ were responsible for the study design, conception. SC and JH were responsible for data collection. BD and AK were responsible for the majority of data analysis and writing of the results sections. $\mathrm{SC}$ was responsible for writing and editing the rest of the document. All authors accept responsibility for the paper.

\section{Details of Ethics Approval}


IRB Status: The institutional review board approved this study (IRB HM2004659) as exempt on March 3 2018 as it was a review of already existing information and posed minimal/no risk to subjects.

\section{Funding}

Services supporting this research project were generated by the VCU Massey Cancer Center Biostatistics Shared Resource, supported, in part, with funding from NIH-NCI Cancer Center Support Grant P30 CA016059.

This paper was presented at the 2019 ACOG Annual Clinical and Scientific Meeting at the Nashville Music City Center in Nashville, TN, May 3-6, 2019

\section{References}

1. Cervical Cancer | CDC [Internet]. [cited 2020 Aug 6]. Available from: https://www.cdc.gov/cancer/cervical/index.htm

2. Screening Guidelines - ASCCP [Internet]. [cited 2020 Aug 6]. Available from: https://www.asccp.org/screening-guidelines

3. Ho GYF, Bierman R, Beardsley L, Chang CJ, Burk RD. Natural History of Cervicovaginal Papillomavirus Infection in Young Women. N Engl J Med. 1998 Feb 12;338(7):423-8.

4. 2012 Updated Consensus Guidelines for the Management of Abnormal Cervical Cancer Screening Tests and Cancer Precursors: Erratum. J Low Genit Tract Dis. 2013 Jul;17(3):367.

5. Bruno MT, Ferrara M, Fava V, Rapisarda A, Coco A. HPV genotype determination and E6/E7 mRNA detection for management of HPV positive women. Virol J. 2018 Mar 27;15(1).

6. Perkins RB, Guido RS, Castle PE, Chelmow D, Einstein MH, Garcia F, et al. 2019 ASCCP Risk-Based Management Consensus Guidelines for Abnormal Cervical Cancer Screening Tests and Cancer Precursors. J Low Genit Tract Dis. 2020 Apr;24(2):102-131.

7. Teoh DGK, Marriott AE, Isaksson Vogel R, Marriott RT, Lais CW, Downs LS, et al. Adherence to the 2012 national cervical cancer screening guidelines: a pilot study. Am J Obstet Gynecol. 2015 Jan;212(1):62.e1-9.

8. Cappiello JD, Boardman M. Longitudinal study of advanced practice nurses' implementation of screening intervals for cervical cancer screening: J Am Assoc Nurse Pract. 2018 Feb;30(2):92-100.

9. Christopherson WM, Lundin FE, Mendez WM, Parker JE. Cervical cancer control: a study of morbidity and mortality trends over a twenty-one-year period. Cancer. 1976 Sep;38(3):1357-66.

10. Walboomers JM, Jacobs MV, Manos MM, Bosch FX, Kummer JA, Shah KV, et al. Human papillomavirus is a necessary cause of invasive cervical cancer worldwide. J Pathol. 1999 Sep;189(1):12-9.

11. Jakobsson M, Gissler M, Paavonen J, Tapper A-M. Loop electrosurgical excision procedure and the risk for preterm birth. Obstet Gynecol. 2009 Sep;114(3):504-10.

12. Committee on Practice Bulletins - Gynecology. Practice Bulletin No. 168: Cervical Cancer Screening and Prevention. Obstet Gynecol. 2016;128(4):e111-130.

\section{Tables}

Table 1 Descriptive Statistics for 2012 ASCCP Guideline Adherence

All Referrals $(n=430)$ Disco

\begin{tabular}{ll}
\hline Appropriate Referral & \\
No & $75(17.4)$ \\
Yes & $355(82.6)$ \\
Pap Result &
\end{tabular}




\begin{tabular}{|c|c|c|}
\hline & All Referrals $(n=430)$ & Discc \\
\hline NILM & $39(9.07)$ & $15(20$ \\
\hline ASCUS & $123(28.6)$ & $22(29$ \\
\hline $\mathrm{ASCH}$ & $35(8.1)$ & $0(0)$ \\
\hline LSIL & $180(41.8)$ & $36(48$ \\
\hline HSIL & $48(11.1)$ & $2(2.7)$ \\
\hline $\mathrm{AGC}$ & $5(1.1)$ & $0(0)$ \\
\hline \multicolumn{3}{|c|}{ Recommendation by Reviewing Attending } \\
\hline Colposcopy & $340(79.1)$ & $23(6.8$ \\
\hline LEEP & $34(7.9)$ & $1(2.9)$ \\
\hline Re-Pap & $56(13)$ & $51(91$ \\
\hline \multicolumn{3}{|c|}{ Recommendation Appropriate (reviewing attending) } \\
\hline No & $24(5.5)$ & $21(28$ \\
\hline Yes & $406(94.4)$ & $54(72$ \\
\hline \multicolumn{3}{|c|}{ Post-Colposcopy Recommendation Guideline Adherence by VCU Physician } \\
\hline Yes & $309(71.8)$ & $20(26$ \\
\hline No & $18(4.2)$ & $6(8)$ \\
\hline $\mathrm{N} / \mathrm{A}$ & $103(24.0)$ & $49(65$ \\
\hline \multicolumn{3}{|l|}{ Clinic Type } \\
\hline OBGYN & $53(12.3)$ & $6(8)$ \\
\hline Internal Medicine & $22(5.1)$ & $9(12)$ \\
\hline Family Medicine & $87(20.2)$ & $30(40$ \\
\hline $\mathrm{VA}$ & $42(9.8)$ & $3(4)$ \\
\hline Health District & $193(44.9)$ & $21(28$ \\
\hline Others & $33(7.7)$ & $6(8)$ \\
\hline \multicolumn{3}{|l|}{ HPV Result } \\
\hline Positive & $233(54.2)$ & $32(42$ \\
\hline Negative & $32(7.4)$ & $14(18$ \\
\hline $\mathrm{N} / \mathrm{A}$ & $165(38.4)$ & $29(38$ \\
\hline \multicolumn{3}{|l|}{ Prior Colposcopy } \\
\hline Yes & $139(32.3)$ & $10(13$ \\
\hline No & $178(41.4)$ & $30(40$ \\
\hline Unknown & $113(26.2)$ & $35(46$ \\
\hline \multicolumn{3}{|l|}{ VCU Clinic Visit } \\
\hline Yes & $313(72.8)$ & $23(30$ \\
\hline No & $61(14.2)$ & $8(10.7$ \\
\hline Referred Back to Provider & $51(11.9)$ & $44(58$ \\
\hline \multicolumn{3}{|l|}{ Race } \\
\hline White & $135(31.4)$ & $12(16$ \\
\hline African American & $219(50.9)$ & $49(65$ \\
\hline Hispanic & $47(10.9)$ & $6(8)$ \\
\hline Other/Unknown & $29(6.74)$ & $8(10.7$ \\
\hline \multicolumn{3}{|l|}{ Age Group } \\
\hline $21-24$ & $53(12.3)$ & $31(41$ \\
\hline $25-29$ & $122(28.4)$ & $5(6.7)$ \\
\hline $30-64$ & $255(59.3)$ & $39(52$ \\
\hline
\end{tabular}

${ }^{* * *} \mathrm{p}<0.001,{ }^{* *} \mathrm{p}<0.01,{ }^{*} \mathrm{p}<0.05$

Table 2 Descriptive Statistics for Discordant Referrals According to Cervical Cytology Results and ASCCP Age Group Recommendations 


\begin{tabular}{|c|c|c|c|c|c|c|c|}
\hline Age Group & 21-24 & $21-24$ & 21-24 & $25-29$ & $25-29$ & $30-64$ & 30 \\
\hline \multirow[t]{2}{*}{ Number of patients within each age group } & 31 & 31 & 31 & 5 & 5 & 39 & 00 \\
\hline & ASCUS & LSIL & NILM & ASCUS & NILM & ASCUS & $\mathbf{L S}$ \\
\hline Inappropriate Referral $(\mathrm{N}=75)$ & $6(19.4)$ & $24(77.4)$ & $1(0.3)$ & $1(0.2)$ & $4(0.8)$ & $15(38)$ & \\
\hline Recommendation & $p$-value $=$ & 0.8236 & & p-value $=$ & 0.0253 & p-value $=$ & \\
\hline Colposcopy & $1(16.7)$ & $8(33.3)$ & $0(0)$ & $1(100)$ & $0(0)$ & $3(20)$ & \\
\hline LEEP & $0(0)$ & $1(4.2)$ & $0(0)$ & 0() & 0() & 0() & 0() \\
\hline Re-Pap & $5(83.3)$ & $15(62.5)$ & $1(100)$ & $0(0)$ & $4(100)$ & $12(80)$ & \\
\hline Recommendation Appropriate & $p$-value $=$ & 0.4854 & & p-value $=$ & 0.0253 & p-value $=$ & \\
\hline No & $1(16.7)$ & $9(37.5)$ & $0(0)$ & $1(100)$ & $0(0)$ & $3(20)$ & \\
\hline Yes & $5(83.3)$ & $15(62.5)$ & $1(100)$ & $0(0)$ & $4(100)$ & $12(80)$ & \\
\hline Clinic Type & $p$-value $=$ & 0.4009 & & p-value $=$ & 0.6592 & $p$-value $=$ & \\
\hline OB & $2(33.3)$ & $2(8.3)$ & $0(0)$ & $0(0)$ & $0(0)$ & $0(0)$ & \\
\hline IM & $0(0)$ & $0(0)$ & $0(0)$ & $0(0)$ & $0(0)$ & $6(40)$ & \\
\hline FM & $3(50)$ & $6(25)$ & $1(100)$ & $0(0)$ & $1(25)$ & $8(53.3)$ & \\
\hline VA & $0(0)$ & $1(4.2)$ & $0(0)$ & $0(0)$ & $0(0)$ & $1(6.7)$ & \\
\hline $\mathrm{HD}$ & $0(0)$ & $12(50)$ & $0(0)$ & $1(100)$ & $2(50)$ & $0(0)$ & \\
\hline Others & $1(16.7)$ & $3(12.5)$ & $0(0)$ & $0(0)$ & $1(25)$ & $0(0)$ & \\
\hline Prior Colposcopy & $p$-value $=$ & 0.1959 & & p-value $=$ & 0.0821 & p-value $=$ & \\
\hline Yes & $1(16.7)$ & $0(0)$ & $0(0)$ & $0(0)$ & $2(50)$ & $2(13.3)$ & \\
\hline No & $3(50)$ & $10(41.7)$ & $1(100)$ & $1(100)$ & $0(0)$ & $6(40)$ & \\
\hline $\mathrm{N} / \mathrm{A}$ & $2(33.3)$ & $14(58.3)$ & $0(0)$ & $0(0)$ & $2(50)$ & $7(46.7)$ & \\
\hline Race Ethnicity & $p$-value $=$ & 0.5809 & & $p$-value $=$ & 0.6592 & $p$-value $=$ & \\
\hline White & $1(16.7)$ & $1(4.2)$ & $0(0)$ & $0(0)$ & $0(0)$ & $3(20)$ & \\
\hline African American & $4(66.7)$ & $15(62.5)$ & $1(100)$ & $1(100)$ & $3(75)$ & $11(73.3)$ & \\
\hline Hispanic & $1(16.7)$ & $1(4.2)$ & $0(0)$ & $0(0)$ & $1(25)$ & $1(6.7)$ & \\
\hline Other & $0(0)$ & $7(29.2)$ & $0(0)$ & $0(0)$ & $0(0)$ & $0(0)$ & \\
\hline
\end{tabular}

${ }^{* * *} \mathrm{p}<0.001,{ }^{* *} \mathrm{p}<0.01,{ }^{*} \mathrm{p}<0.05$

Table 3 Univariate and Multivariable Logistic Regression Models for Concordant/Discordant Referrals

\begin{tabular}{|c|c|c|c|}
\hline Appropriate Referral & Odds Ratio (95\% CI) & P-Value & Odds Ratio (95\% CI \\
\hline Pap Result $($ Ref=Low;n=430) & Pap Result $($ Ref $=$ Low;n=430) & & \\
\hline High & $0.75(0.58,0.96)$ & $*$ & $0.03(0.007,0.17)$ \\
\hline Clinic Type $($ Ref $=$ OBGYN; $n=430)$ & Clinic Type $($ Ref $=$ OBGYN; $n=430)$ & & \\
\hline FM & $4.12(1.58,10.74)$ & $* *$ & $7.72(2.23,26.69)$ \\
\hline HD & $0.95(0.36,2.5)$ & 0.9277 & $1.58(0.46,5.39)$ \\
\hline IM & $5.42(1.63,18.03)$ & 0.0058 & $10.94(2.56,46.79)$ \\
\hline VA & $0.6(0.14,2.56)$ & 0.4937 & $1.16(0.21,6.4)$ \\
\hline Other & $1.74(0.51,5.93)$ & 0.3758 & $3.79(0.74,19.32)$ \\
\hline Race $($ ref $=$ white $; n=396)$ & Race $($ ref $=$ white $; n=396)$ & & \\
\hline African American & $2.95(1.5,5.78)$ & 0.0747 & $2.25(0.99,5.1)$ \\
\hline Hispanic & $1.5(0.52,4.25)$ & 0.3859 & $1.21(0.34,4.26)$ \\
\hline Other/Unknown & & & \\
\hline
\end{tabular}

\section{Hosted file}

Figure 1.pdf available at https://authorea.com/users/384886/articles/500432-adherence-tothe-american-society-for-colposcopy-and-cervical-pathology-guidelines-an-observational- 
study 